\title{
Effects of Transplanting Bone Marrow Stromal Cells Transfected with CXCL13 on Fracture Healing of Diabetic Rats
}

\author{
Hui Jiang Yicun Wang Jia Meng Shuo Chen Jun Wang Yang Qiu \\ Jianning Zhao Ting Guo \\ Department of Orthopedics, Jinling Hospital, Clinical School of Nanjing, Second Military Medical \\ University, Nanjing, Jiangsu, China
}

\section{Key Words}

Diabetes mellitus $\cdot$ Fracture healing $\cdot$ BMSCs $・$ CXCL13

\begin{abstract}
Background/Aims: Diabetic fracture have poor treatment and serious complications. Therefore, how to treat diabetic fracture is receiving increasing attention. This study aimed to investigate the effects of transplanting CXCL13-stimulated bone marrow stromal cells (BMSCs) on the fracture healing in diabetic rats. Methods: In vitro, RT-PCR was employed to examine the expression of CXCL13 in BMSCs in high glucose environment. MTT assay and apoptosis assay were utilized to determine the effects of CXCL13 overexpression on the proliferation and apoptosis of BMSCs respectively. ALP staining was applied to detect the ALP activity. In vivo, CXCL13-stimulated BMSCs were transplanted into the fracture sites of diabetic rats. At the $1^{\text {st }}$ week, $2^{\text {nd }}$ weeks, $4^{\text {th }}$ week and $6^{\text {th }}$ week after the operation, bone mineral density (BMD) and callus area measurement, ELISA detection, and HE staining were performed to evaluate the fracture healing. Results: Low BMD and less area of callus in diabetic rats showed that the recovery after fracture was worse in diabetic rats than in non-diabetic rats. Meanwhile, the expression of CXCL13 in serum was lower in diabetic rats than in non-diabetic rats. Overexpression of CXCL13 promoted the proliferation of BMSCs in vitro high glucose environment. After BMSCs transfected with CXCL13 being transplanted into the fracture sites of diabetic rats, it was found that the fracture healing was enhanced and ALP expression in serum became higher. HE staining results further verified the effects of transplantation of BMSCs transfected with CXCL13 on fracture healing in diabetic rats. Conclusion: These finding indicated that CXCL13 may play a critical role in the process of fracture healing, which could provide a deeper insight into molecular targets for the fracture healing in diabetic people.
\end{abstract}

$\mathrm{H}$. Jiang and $\mathrm{Y}$. Wang contributed equally to this work.

Jianning Zhao

and Ting Guo 


\section{Cellular Physiology Cell Physiol Biochem 2018;49:123-133 \begin{tabular}{ll|l} 
DOI: 10.1159/000492848 & $\begin{array}{l}\text { O 2018 The Author(s). Published by S. Karger AG, Basel } \\
\text { www.karger.com/cpb }\end{array}$ \\
\hline
\end{tabular} \\ Jiang et al.: CXCL13 Promoted the Fracture Healing in Diabetic Rats}

\section{Introduction}

Diabetes mellitus (DM) is a widespread disease that characterized by hyperglycemia. More than 300, 000, 000 people are suffering from DM and the number of DM patients is still on the rise [1]. Until 2030, it is estimated that about $439,000,000$ people may be affected by DM worldwide [2]. The complications of DM are various, including kidney failure, diabetic ketoacidosis, cardiovascular disease, foot ulcers, and tissue and organ damage [1]. Among them, chronic inflammation is generally regarded as the critical one [3]. Several studies have reported that the reduced bone formation, osteopenia and osteoporosis often occurred in diabetic patients, which induced the impaired fracture healing in diabetic patients $[2,4,5]$. Many stressful diabetic patients were reported to suffer rising blood glucose, ketoacidosis and electrolyte disturbance after the fracture [6]. Furthermore, delayed healing, nonunion, nerve palsy and muscle atrophy were also the adverse consequences occurred in diabetic patients with fracture [7-9]. The life quality and economy of the patients were also affected by the incision infection, osteomyelitis and other serious consequences caused by the delayed fracture healing [10]. With the development of cell biology and molecular biology, the role of cytokines that affected bone metabolism during the fracture healing in diabetic patients has been paid more and more attention $[11,12]$.

Fracture healing is a regenerative process, including coordinated activity of inflammatory cells, chemokines, chondrocytes, osteoblasts and other cell types $[3,13]$. Increasing studies have focused on the process of fracture. W Zhou et al.'s study suggested that endogenous PTH enhanced BMPR2 expression and then accelerated the process of fracture healing [14]. Some other researchers unveiled that macrophages and osteoblastic cell played a functional role in the fracture healing $[15,16]$. Here, we focused on the bone marrow stromal cells (BMSCs) treatment. The BMSCs, also called bone mesenchymal stem cells (BMSCs), are stem cells with capacities of multi-differentiation and were widely applied to organ repair and cell therapy $[17,18]$. It has been reported that BMSCs can differentiate to osteoblasts, chondrocytes osteoblasts and adipocytes $[3,18]$. Therefore, BMSCs play important roles in the tissue regeneration, and the application of BMSCs for the tendon-bone healing and fracture healing is quite promising $[18,19]$. Being induced to the injured sites, BMSCs can reduce the inflammation and promote the angiogenesis [3].

The function of these small cytokine-like proteins was reported to be related to their seven transmembrane-domain glycoprotein receptors which are coupled to the $G$ protein coupled receptor [20]. As a member of the chemokines, CXC chemokine ligand-13 (CXCL13) and its receptors were invloved in the process of BMSCs migration. For example, CXCL13 together with chemokine receptor-5 (CXCR5) regulated the B-cell chemotaxis and the recruitment of BMSCs during the fracture healing [18, 21,22]. Further studies indicated that the inflammatory cytokine $I L-6$ can induced the expression of CXCL13 in osteoblast, which implied the possible important role of CXCL13 during the fracture healing [18].

While the role of CXCL13 and BMSCs in promoting fracture healing has been extensively researched, their functions in DM fracture healing are much less known. A prior research of KI Ko et al. suggested that the diabetes-enhanced Tumor-Necrosis-Factor-alpha (TNF- $\alpha$ ) reduces BMSCs during fracture healing [3]. Another study conducted by Granero-Molto et al. showed that transplantation of BMSCs improved the fracture mechanical strength and increased new bone content [23]. The purpose of this study is to determine the role of CXCL13 in fracture healing by analyzing BMSC transfected with CXCL13 in vitro as well as to explore the effects of transplantation of BMSCs transfected with CXCL13 in DM and non-DM femur fracture model. 


\section{Cellular Physiology Cell Physiol Biochem 2018;49:123-133 \begin{tabular}{ll|l} 
and BiOChemistry & $\begin{array}{l}\text { DOI: 10.1159/000492848 } \\
\text { Published online: 22 August, } 2018\end{array}$ & $\begin{array}{l}\text { (c) } 2018 \text { The Author(s). Published by S. Karger AG, Basel } \\
\text { www.karger.com/cpb }\end{array}$ \\
\hline
\end{tabular}}

\section{Materials and Methods}

\section{Animals}

Ninety outbred male Wistar rats (clean grade, age: 7-8 weeks, weight: 210-230g) were provided by the Jinling Hospital, Nanjing, China. All rats were allowed to acclimatize to a condition with room temperature $18^{\circ} \mathrm{C}-20^{\circ} \mathrm{C}$ and relative humidity $40 \%-60 \%$. The experiments were performed one week after the feeding. Our research had been approved by the Jinling Hospital as well.

\section{DM fracture model}

72 rats were divided into three groups at random with 24 rats in each group. One is the non-DM group and the other two are the diabetic groups (24 rats in the DM group and 24 rats in the BMSC treatment group). The rat DM model induced by streptozotocin (STZ, Sigma-Aldrich, St. Louis, MO, USA) was performed as previously described [3]. In brief, all rats in the diabetic group were intraperitoneally injected with STZ as $40 \mathrm{mg}$ per body mass and fed with ordinary fodder for 7 days. Caudal vein blood was collected to detect the blood glucose. The rats were considered to be diabetic if blood glucose $>16.7 \mathrm{mmol} / \mathrm{L}$. After the establishment of diabetic rat model, all rats were subjected to general anesthesia so as to establish a fracture model. The incision was aseptically made on the left leg and the superior segments of tibia were then exposed. The tibia was sawed off 1-2 cm below the tibia plateau. After a 10-min iodine immersion, the incision was sutured and fixed with a splint, and the rats were fed separately.

Bone mineral density (BMD) and maximum cross-sectional area of callus

At the $1^{\text {st }}, 2^{\text {nd }}, 4^{\text {th }}$ and $6^{\text {th }}$ weeks after the operation, 6 rats were picked from each group randomly and then executed. BMD changes of the $2.0 \mathrm{~mm} \times 1.5 \mathrm{~mm}$ rectangular region centered on the fracture were detected through a dual-energy X-ray absorptiometry (Lunar DPX-NT, General Electric Company, New York, USA) by using fast scanning mode. Then we removed the soft tissue around the fracture completely, measured the maximum diameter of the callus, and calculated the maximum cross-sectional area.

\section{Serum and tissue samples}

For serum samples, $2 \mathrm{~mL}$ portal venous blood was collected and centrifuged at $3000 \mathrm{rpm}$ for $15 \mathrm{~min}$. The supernatant was transferred into Eppendorf tube and then stored at $-20^{\circ} \mathrm{C}$ until ready for use. For histological detection, bone tissues close to the center of fracture site were washed with phosphate buffer saline (PBS) and placed in 10\% neutral formalin. Having been fixed at room temperature for $24 \mathrm{~h}$, the tissue samples were decalcified in 15\% EDTA-2Na solution, dehydrated in gradient ethanol and then embedded in paraffin. Ultimately, the tissues samples were sectioned for histological detection.

\section{BMSC isolation and culture}

Femur was obtained from Wistar rats for primary culture of BMSCs. Briefly, all rats were sacrificed and $3 \mathrm{~mL}$ marrows were extracted by using injection syringe containing $2 \mathrm{~mL}$ heparin anticoagulation. BMSCs were prepared after a 1000-rpm centrifugation for $30 \mathrm{~min}$ and then cultured in DMEM medium containing $10 \%$ fetal bovine serum (FBS) at $37^{\circ} \mathrm{C}$.

\section{BMSCs identification}

BMSCs were harvested and plated into 24-well plates at a density of $1 \times 10^{5}$ cells $/ \mathrm{mL}$. After the overnight incubation, the cells were fixed in 4\% paraformaldehyde (Beyotime, Shanghai, China), blocked with confining liquid and washed with PBS. The phycoerythrin (PE)-conjugated primary antibodies against CD34, CD29 and CD73 (cloud-clone, Wuhan, China) were added to cells. Then the BMSCs were detected by using a flow cytometer (Becton, Dickinson and Company, Franklin Lakes, NJ, USA). Isotype control antibodies were used as the negative control.

\section{In vitro experiments}

The in vitro experiments were carried out in three groups: the normal group, the high-glucose (HG) group and the HG+CXCL13 group. In normal group, the BMSCs were seeded in normal culture (glucose concentration: $6.5 \mathrm{mmol} / \mathrm{L}$ ). In HG and HG+CXCL13 groups, BMSCs were cultured in the DMEM medium with a glucose concentration $30 \mathrm{mmol} / \mathrm{L}$. All BMSCs in different groups were cultivated in a humidified incubator 


\section{Cellular Physiology Cell Physiol Biochem 2018;49:123-133 \begin{tabular}{ll|l} 
DOI: 10.1159/000492848 & $\begin{array}{l}\text { O 2018 The Author(s). Published by S. Karger AG, Basel } \\
\text { www.karger.com/cpb }\end{array}$ \\
\hline
\end{tabular}}

Jiang et al.: CXCL13 Promoted the Fracture Healing in Diabetic Rats

at $37^{\circ} \mathrm{C}$ BMSCs in $\mathrm{HG}+\mathrm{CXCL13}$ group were transfected with CXCL13. Briefly, the CXCL13 were cloned into the pAD/CMV/ V5-DEST vectors (Invitrogen, CarIsbad, CA, USA) according to the manufacturer's instructions. The prepared CXCL13overexpression vector and liposome2000 (Invitrogen) were then dissolved in DMEM medium. After 30-minute standing, the solution was added into BMSCs to establish the CXCL13-overpression BMSCs.

The apoptosis assay was performed by using a PI Annexin V Apoptosis Detecting Kit (BD, Franklin Lakes, NJ, USA) following the manufacturer's instructions at the $10^{\text {th }}$ day after the transfection. Then the FACS Calibur (BD) was applied to detect the cell apoptosis and the experimental data were analyzed by FACS Diva (BD).

\section{ALP staining}

ALP staining was conducted on the cell populations treated and cultured for 10 days. The medium was removed, and the cells were incubated at room temperature on a shaker with ALP staining solution for 30 min after fixed with 4\% paraformaldehyde. Afterwards, the ALP staining solution was discarded, and cells were covered with coverslip. The cell slides were observed under a light microscopy at a magnification of $100 \times$.

\section{Real-time PCR (RT-PCR)}

In accordance with the instructions, we extracted total RNA by uisng Trizol reagent (Invitrogen, USA). The concentration of total RNA was analyzed with the spectrophotometer (Beckham, USA). Revert AidFirst Strand cDNA Synthesis Kit (Fermentas, USA) was used to reversely transcribe RNA into complementary DNA (cDNA). The expressions of CXCL13 and $\beta$-actin were examined by RT-PCR, and the primers were shown in Table 1. MRNA expression was quantified by using the $2^{-\Delta t}$ method with $\beta$-actin as an endogenous control.

\section{Western blot}

The proteins extracted from BMSCs were quantified by Bradford method. Then these protein samples were separated by Sodium dodecylsulphate polyacrylamide gel electrophoresis (SDS-PAGE). After blocking the Polyvinylidene Fluoride (PVDF) membranes in 5\% skim milk (resolved in TBYS buffer) for $2 \mathrm{~h}$, primary antibodies for CXCL13 (ab112521, 1:100, Abcam, Cambridge, MA, USA), $\beta$-actin (ab8226, 1:2500, Abcam) and secondary antibodies (ab6721, 1:2000, Abcam) were incubated with the membranes at $4^{\circ} \mathrm{C}$ overnight and $37^{\circ} \mathrm{C} 1 \mathrm{~h}$ respectively. The membranes were detected by means of the chemiluminescence and Lab Works 4.5 image acquisition and analysis system. $\beta$-actin was considered as an internal control here.

\section{ELISA}

Quantitative analysis of ALP and CXCL13 concentration was respectively conducted by using ALP (alkaline phosphatase) ELISA kit (GRDS13-355, Shanghai Guang Rui Biological Technology Co., Ltd, Shanghai, China) and CXCL13 ELISA kit (PRCU6435, Prajna Research, Beijing, China) following the manufacturer's instructions. Briefly, a $25 \mu \mathrm{L}$ prepared serum sample was mixed with a $25 \mu \mathrm{L}$ freshly prepared paranitrophenyl phosphate and incubated at $37^{\circ} \mathrm{C}$ for $30 \mathrm{~min} .100 \mu \mathrm{L} 0.2 \mathrm{~mol} / \mathrm{L} \mathrm{NaOH}$ was added to terminate the enzymatic reaction. The absorbance was determined by means of an enzyme-linked immunosorbent assay reader (Multiskan MK3, Thermo Fisher Scientific, Waltham, MA, USA) at optical density (OD) $405 \mathrm{~nm}$ (for ALP) and $450 \mathrm{~nm}$ (for CXCL13).

\section{MTT assays}

BMSCs were inoculated in a 96 -well plate at a density of $1 \times 10^{4}$ per well. At the $1^{\text {st }}, 3^{\text {rd }}, 5^{\text {th }}, 7^{\text {th }}$ and $10^{\text {th }}$ day after cell culture, BMSCs were supplemented with the MTT solution $(10 \mu \mathrm{L}$, Sigma-Aldrich, St. Louis, MO, USA) and incubated for $4 \mathrm{~h}$ to detect the cell proliferation. $150 \mu \mathrm{L}$ DMSO (Sigma) was then added to dissolve the crystal substance. The absorbance was measured at $490 \mathrm{~nm}$ by using the enzyme-linked 


\section{Cellular Physiology Cell Physiol Biochem 2018;49:123-133 \begin{tabular}{l|l|l} 
DOI: 10.1159/000492848 & $\begin{array}{l}\text { O } 2018 \text { The Author(s). Published by S. Karger AG, Basel } \\
\text { wwww.karger.com/cpb }\end{array}$
\end{tabular}

Jiang et al.: CXCL13 Promoted the Fracture Healing in Diabetic Rats

immunosorbent assay reader. The growth curve was plotted with the incubation time as the abscissa and OD value as the ordinate.

\section{Hematoxylin and eosin (HE) staining}

Prepared specimen was dewaxed with xylene and then dehydrated by using the gradient ethanol. Subsequently, the tissue sections were stained in hematoxylin solution for $10 \mathrm{~min}$. Having been washed with water for $1 \mathrm{~h}$, the tissue sections were dehydrated with $70 \%$ and $90 \%$ alcohol for $10 \mathrm{~min}$ respectively and stained in eosin solution for $3 \mathrm{~min}$. The dehydrated sections were observed under a light microscopy at a magnification of 200x.

\section{In vivo experiments}

In vivo experiments were divided into three groups as follows: the non-DM group, the DM group and the treatment group. DM fracture model used for the BMSCs treatment was established in accordance with the above methods. Having been transfected with lentivirus of overexpression of CXCL13, BMSCs were washed wish PBS and adjusted to the concentration of $2 \times 10^{6} / \mathrm{mL} .0 .4 \mathrm{~mL}$ BMSCs were injected into the fracture sites 3 days after the fracture model building. Similarly, BMD and the maximum cross-sectional area were measured at the $1^{\text {st }}, 2^{\text {nd }}, 4^{\text {th }}, 6^{\text {th }}$ week after establishing the treatment group. Fracture healing was observed through HE staining and expression of CXCL13 in the serum was measured by RT-PCR.

\section{Statistical analysis}

GraphPad Prism 7.0 software (GraphPad Software, USA) was used for statistical analysis and data was expressed as mean \pm standard deviation. Data were compared by unpaired $t$-test (differences between two groups) or oneway ANOVA analysis (differences among groups). $P<0.05$ was considered to be statistically significant.

\section{Results}

\section{Fracture healing in DM rats}

At the $1^{\text {st }}, 2^{\text {nd }}, 4^{\text {th }}, 6^{\text {th }}$ week after the fracture, we detected the changes of BMD and maximum cross-sectional area of callus in each group. As shown in Fig. 1A and Fig. $1 \mathrm{~B}$, no significant difference was found between two groups at the first two weeks $(P>0.05)$. With increasing time, the difference became more conspicuous. The BMD was lower and the maximum cross-sectional area was larger in DM group than in non-DM group at the $4^{\text {th }}$ and $6^{\text {th }}$ week after the fracture $(P<0.05)$. HE staining results also showed the poor healing after the fracture in DM rats. One week after the operation, we can clearly detect cell infiltration, chondrocytes, granulation tissue and angiogenesis in non-DM group. While in the DM group, there were mainly red blood cells, inflammatory

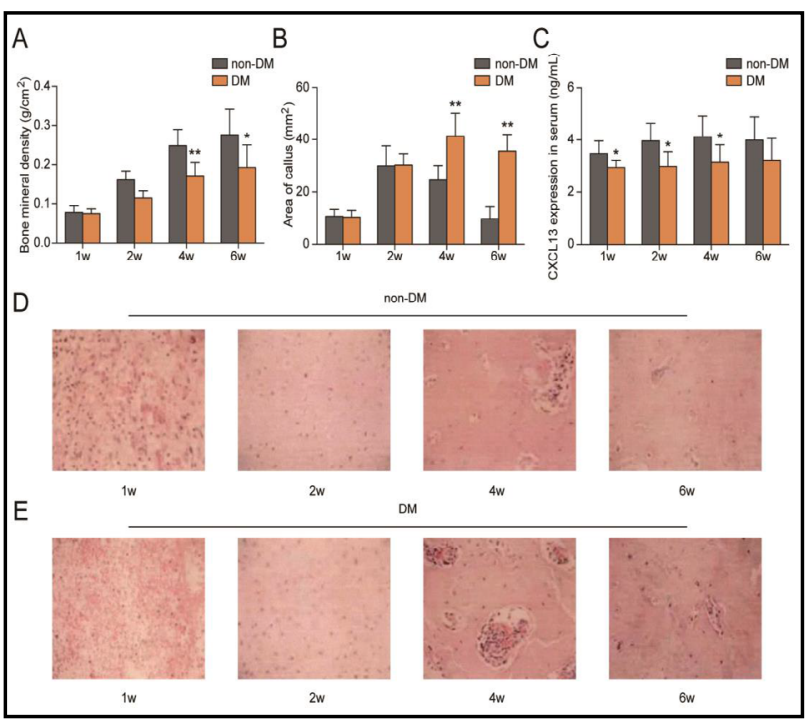

Fig. 1. The differences between the process of fracture healing in normal rats and diabetic rats A: DM rats had lower BMD during the fracture healing. ${ }^{*} \mathrm{P}<0.05$, ${ }^{* *} \mathrm{P}<0.01$, compared with the non-DM group. DM: Diabetes mellitus. B: Callus area changes at different times after fracture in nonDM group and diabetic group. ${ }^{* *} \mathrm{P}<0.01$, compared with the non-DM group. DM: Diabetes mellitus. C: CXCL13 ELISA kit was used to detected CXCL13 concentration in non-DM group and diabetic group. CXCL13 expression was lower in DM group. ${ }^{*} \mathrm{P}<0.05$, compared with the non-DM group. DM: Diabetes mellitus. D: HE staining results for bone callus in non-DM group at different times (magnification: $\times 200$ ). E: HE staining results for bone callus in DM group at different times (magnification: $\times 200$ ). 
Fig. 2. Effects of CXCL13 on the proliferation of BMSCs in the high glucose environment. A: Detection of the antigen profiles on the cell surface of human BMSCs by flow cytometry analysis. BMSCs were CD29 and CD73 positive $\quad(100 \%$ respectively) and negative for CD34 (13.4\% respectively). B-C: The expression of CXCL13 was measured by RTPCR (B) and western blot (C), CXCL13 was high-expressed in the normal environment and lowexpressed in HG environment. $* \mathrm{P}<0.05$, compared with normal environment, \#\# $\mathrm{P}<0.01$, compared with HG environment. HG: high glucose. D: At 1, 3, 5, 7d and $10 \mathrm{~d}$ of post-transfection, the cell proliferation was measured by MTT assay. Error bars represent mean \pm SD. ${ }^{* *} \mathrm{P}<0.01$, compared with normal environment, $\# \# \mathrm{P}<0.01$, compared with $\mathrm{HG}$ environment. E: At $10 \mathrm{~d}$ of posttransfection, the cell apoptosis rate was analyzed by an apoptosis detecting kit. CXCL13 inhibited

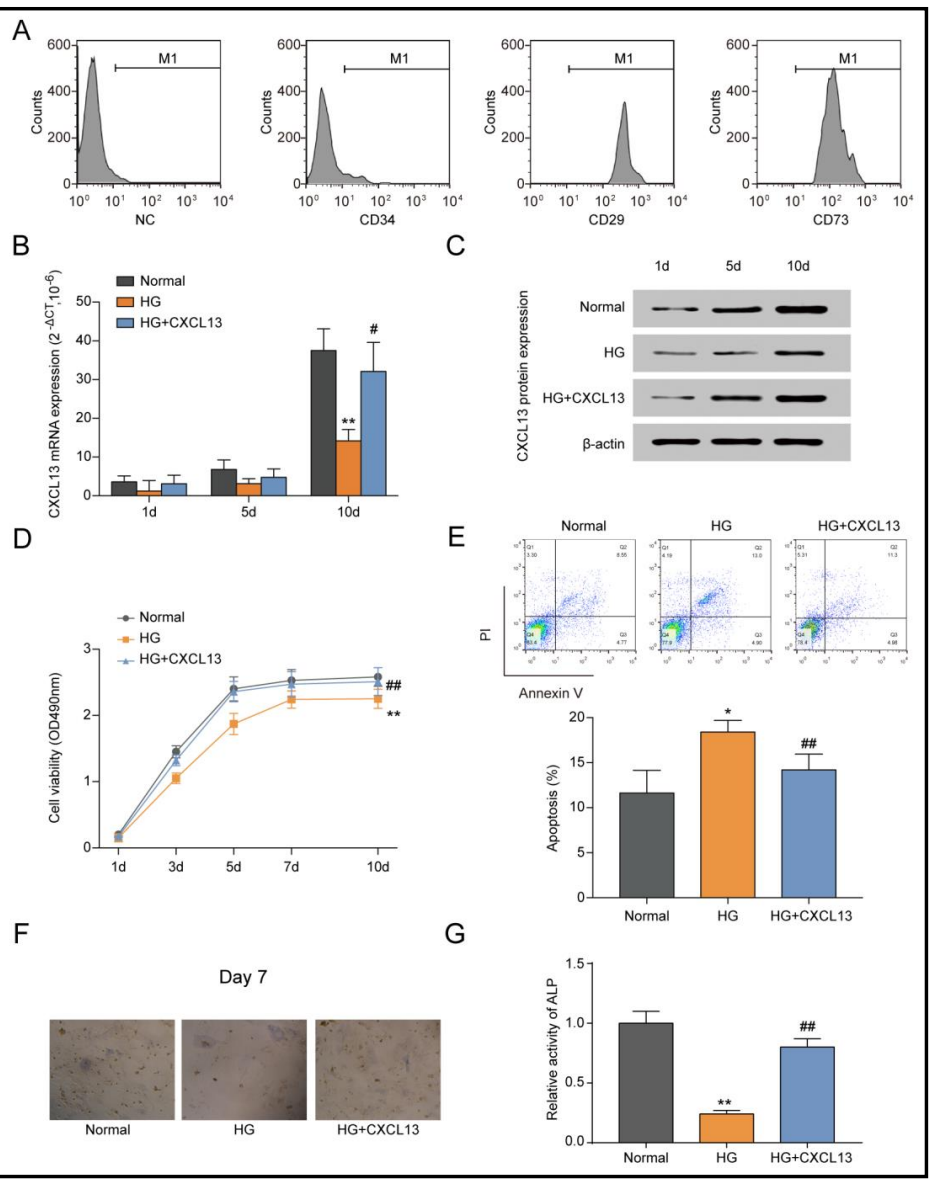
apoptosis of BMSCs in the high glucose environment. ${ }^{*} \mathrm{P}<0.05$, compared with normal environment, \#\#P<0.01, compared with HG environment. F: Differentiation to osteoblasts can be demonstrated by ALP staining. F: Osteoblast-like (ALP-positive and cuboidal in shape) cell fractions were quantified to the control group. ${ }^{* *} \mathrm{P}<0.01$, compared with normal environment, \#\#P<0.01, compared with $\mathrm{HG}$ environment.

cells and fibroblasts (Fig. 1D and Fig. 1E). At the second week, in the non-DM group, the localized mature cartilage cells were deformed and the osteoblasts were arranged on the surface of bone matrix. Inflammatory cells were still visible in DM group, and trabecular bone and woven bone were sparser as well. Four weeks after the fracture, woven callus was found to fuse with each other and the marrow cavity was found larger in non-DM group. In DM group, the new cartilage bone was small and thin, though the cartilage callus area and bone mass increased. At the sixth week, the trabecular bone began to differentiate into lamellar bone in non-DM group. While in DM group, cartilage callus was gradually replaced by new bone trabeculae and there still existed some fibrous callus.

\section{Effects of CXCL13 in vitro experiments}

CXCL13 protein expression in non-DM group and DM group were detected using the CXCL13 ELISA kit. In Fig. 1C, CXCL13 expression was found lower in DM group than in nonDM group at various times ( 1 week, 2 weeks, 4 weeks and 6 weeks, $P<0.05$ ). Thus, in vitro experiments were performed to detect the effects of CXCL13 on BMSCs. Firstly, BMSCs were respectively collected from the femur of rats in non-DM group, the result of flow cytometry analysis showed that BMSCs were CD29 and CD73 positive (100\% respectively) and negative for CD34 (13.4\% respectively) (Fig. 2A). Similarly, the mRNA expression and protein expression of CXCL13 decreased in the high glucose environment (compared with the normal environment, Fig. 2B-C, $P>0.05$ ). As shown in Fig. 2D-E, BMSCs showed 


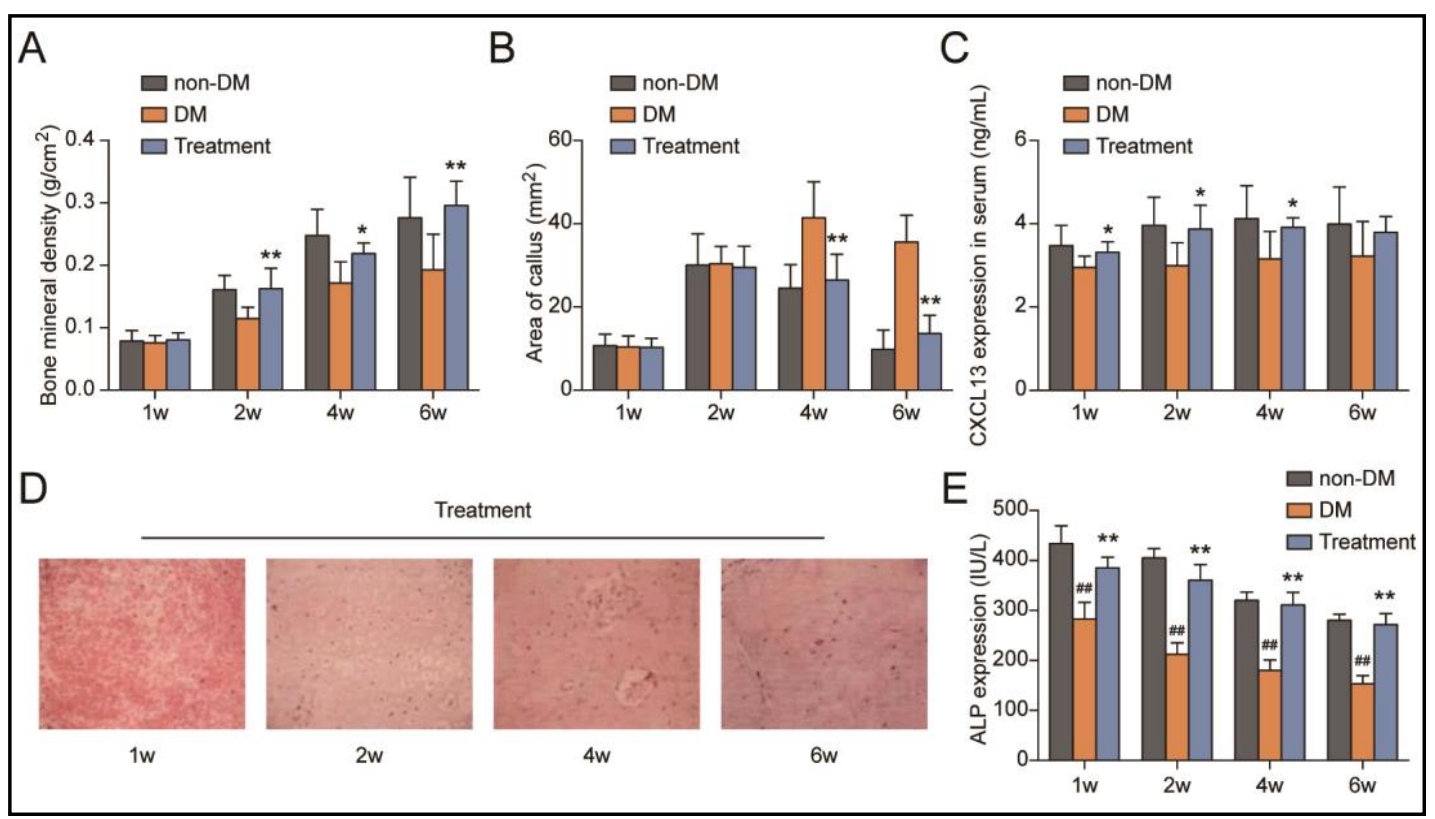

Fig. 3. Effects of CXCL13 on promoting the process of fracture healing in DM group. A-B: BMD values (A) and callus area (B) of different times in treatment group were measured by X-ray absorptiometry, then they were compared with the previous data. ${ }^{* *} \mathrm{P}<0.01$, compared with DM group. C: The concentration of CXCL13 of different times in treatment group was measured by ELISA, the CXCL13 expression was higher in non-DM group and treatment group. ${ }^{*} \mathrm{P}<0.01$, compared with DM group. D: HE staining results for bone callus in treatment group at different times (magnification: $\times 200$ ). E: An ALP ELISA kit was used to detected the ALP expression. ALP was far less in DM group and expression between non-DM group and treatment group was nearly the same. ${ }^{* *} \mathrm{P}<0.01$, compared with $\mathrm{DM}$ group, \#\# $\mathrm{P}<0.01$, compared with non-DM group.

lower proliferation capacity and higher apoptosis rate in the high glucose environment (compared with the normal environment, $P<0.05$ ). The transfection with CXCL13 promoted the proliferation of BMSCs and downregulated the apoptosis rate of BMSCs in the high glucose environment through increasing CXCL13 expressions (Fig. 2B-E). In addition, the histological staining revealed a higher ALP positive rate in BMSCs transfected with CXCL13 in high glucose environment (compared with the normal environment, Fig. $2 \mathrm{~F}-\mathrm{G}, P<0.05$ ). The result demonstrated the differentiation to osteoblasts.

\section{Effects of CXCL13 in vivo experiments}

In vivo animal experiments were performed to further clarify the effects of CXCL13 on proliferation of BMSCs and the fracture healing in DM rats. As shown in Fig. 3A, BMD changes between non-DM group and treatment group were not significant $(P>0.05)$. BMD was always lower in the DM group and the difference between DM group and treatment group was significant $(P<0.05)$. We detected the maximum bone cross-sectional area of callus as well (Fig. 3B). At the first two weeks, the differences were not significant among the three groups. However, at the 4th and 6th week, the maximum cross-sectional area of callus was significantly larger in DM group $(P<0.05)$. The difference between non-DM group and treatment group were not significant $(P>0.05)$. CXCL13 expressions in the serum were confirmed by ELISA (Fig. 3C). The results showed that CXCL13 expression were higher in the treatment group than in the DM group over time $(P<0.05)$. There were mainly red blood cells and inflammatory cells in the fracture site at the first week. At the second week, mature funicular trabeculae were formed with chondrocytes degenerated and osteoblasts arranged on the surface of bone matrix. At the fourth week, marrow cavity became larger and woven 


\section{Cellular Physiology Cell Physiol Biochem 2018;49:123-133 \\ \begin{tabular}{l|l|l} 
DOI: 10.1159/000492848 & $\begin{array}{l}\text { O 2018 The Author(s). Published by S. Karger AG, Basel } \\
\text { www.karger.com/cpb }\end{array}$
\end{tabular} \\ Jiang et al.: CXCL13 Promoted the Fracture Healing in Diabetic Rats}

callus fused with each other to form bridging callus. At the sixth week, the trabecular bone was change to the lamellar bone and the ossification was gradually completed. The results suggested that transplanting BMSCs transfected with CXCL13 was beneficial to the fracture healing in patients with DM.

\section{ALP detection}

The changes of ALP in serum were detected respectively at 1, 2, 4, 6 weeks after the fracture. Over time, ALP decreased in the three groups. ALP activity was significantly lower in DM group than in non-DM group (Fig. $3 \mathrm{E}, P<0.05$ ). In the treatment group, although ALP decreased, the total value was significantly higher than that in the DM group $(P<0.05)$. Difference between non-DM group and treatment was not significant $(P>0.05)$.

\section{Discussion}

Recent studies have focused on promoting the fracture healing in diabetic patients, as delayed union or nonunion often occurred in these patients [24, 25]. Our findings suggested that CXCL13 induced the proliferation of BMSCs in the high glucose environment. Furthermore, we observed that CXCL13-stimulated BMSCs promoted the fracture healing of the diabetic rats, which was substantiated by increased BMD, maximum bone cross-sectional area and enhanced activity of $A L P$.

In the present study, STZ was applied to induce the diabetic fracture model. The results of histological observation also showed that the formation of mature lamellar bone was slower in diabetic group than in the non-DM group, which was in line with previous studies' that bone remodeling can be affected by the diabetes mellitus. One reason for this was the deposition of bone collagen induced by AGEs. Then, osteoblasts can't adhere to collagen and the activities and maturation of osteoclast increased, which resulted in the decrease of BMD and maximum cross-sectional area of callus and may finally become osteoporosis [26, 27]. Our study further confirmed that the bone remodeling was affected by the diabetes mellitus, contributing to the delayed fracture healing.

Bone showed a dramatic ability of regeneration after the injury, and increasing research has found that BMSCs acted as the key factor for the well bone regeneration [28]. As one of the preferred cells for various tissue engineered seed cells, BMSCs exert beneficial influence on the fracture healing $[23,29,30]$. However, diabetes mellitus reduced the BMSCs through various ways. Weinberg et al. reported that diabetes-enhanced advanced glycation end products (AGEs) and the receptor for AGEs (RAGE) inhibited the growth and differentiation of BMSCs [31]. The study of Karnes et al. showed that low expression of TNF- $\alpha$ induced the low expression of BMSCs in diabetic patients [32]. As previously reported, BMSCs are vital to modulate the activity of BMSCs [18, 33]. CXCL13, a member of $C X C$ chemokine family, plays a crucial role in BMSCs [34]. Tian et al. revealed that CXCL13 promotes the effect of MSCs on tendon-bone healing in rats [35]. Another study indicated that CXCL13 induced the proliferation of BMSCs in osteoblasts for bone repair [36]. In our study, we found that BMSCs with a high expression of CXCL13 showed a better proliferation than those with low expression of CXCL13. The in vitro results indicated that CXCL13 promoted the proliferation of BMSCs in high glucose environment, which was consistent with the previous studies.

Then, we injected BMSCs transfected with CXCL13 into the fracture part of diabetic rats for further studies. The expression of CXCL13 in the serum was measured at the $1^{\text {st }}, 2^{\text {nd }}, 4^{\text {th }}$ and $6^{\text {th }}$ week after the treatment. We found that CXCL13 was higher in treatment group than in the other two groups. $A L P$, the alkaline phosphatase, was regarded as the early marker of osteoblast phenotype and differentiation [37,38]. In this study, the increase rate of $A L P$ was significantly lower in diabetic group than in the other two groups at various time points, suggesting a worse osteoblast differentiation in the diabetic group. After treatment with BMSCs transfected with CXCL13, ALP expression in diabetic group increased. The changes of $\mathrm{BMD}$ and maximum cross-sectional area of callus, and the results of histological observation 


\section{Cellular Physiology Cell Physiol Biochem 2018;49:123-133

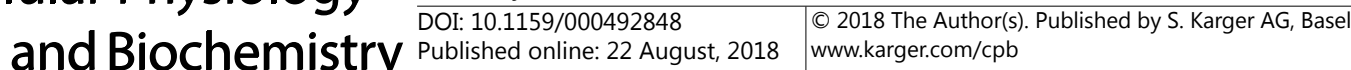 \\ Jiang et al.: CXCL13 Promoted the Fracture Healing in Diabetic Rats}

in the treatment group were similar to those in the non-DM group that CXCL13 can also promote the fracture healing in diabetic rats by promoting the BMSCs' proliferation. Since CXCL13 is involved in the mediation of diabetes, there seems to be another mechanism that CXCL13 regulate the healing of fractures indirectly by regulating diabetes. Thus, more studies should be performed to verify this possible mechanism.

At present, it is necessary for us to find a practical and effective way to treat the diabetic patients with fracture. It was shown in this study that BMSCs transfected with CXCL13 could be used for improving the fracture healing in diabetic patients, which provided a new method for the clinical treatment. However, the effects of CXCL13 on human patients are not clear, which remains to be determined by further studies.

\section{Conclusion}

In summary, this study was the first to show the possible effects of CXCL13 on the proliferation of BMSCs in high glucose environment. We found that diabetes could lead to a reduction in bone mineral density, which seriously affects the fracture healing. In vivo and in vitro experiments showed that CXCL13 promoted the proliferation of BMSCs in high glucose environments, promoting the fracture healing in diabetic rats. Our results might provide a novel insight into the treatment of fracture in diabetic patients.

\section{Abbreviations}

BMSCs (bone marrow stromal cells); CXCL13 (chemokine ligand-13); DM (Diabetes mellitus); CXCR5 (chemokine receptor-5); TNF- $\alpha$ (Tumor-Necrosis-Factor-alpha); BMD (Bone mineral density); FBS (fetal bovine serum); HG (high-glucose); RT-PCR (Real-time PCR); SDS-PAGE (Sodium dodecylsulphate polyacrylamide gel electrophoresis); PVDF (Polyvinylidene Fluoride); OD (optical density); HE (Hematoxylin and eosin); ANOVA (Analysis of Variance); AGEs (advanced glycation end products); RAGE (receptor for AGEs).

\section{Acknowledgements}

This study was supported by the National Nature Science Foundation of China (No. 81702175); Chinese Medical Doctor Association-Special Fund for Thrombosis Prevention after Major Orthopedics Surgery (No. 2015COS0810); Nanjing General Hospital Subject (No. 2017003).

\section{Disclosure Statement}

No conflict of interests exits in the submission of this manuscript and this manuscript has been approved by all authors for publication.

\section{References}

1 Kawser Hossain M, Abdal Dayem A, Han J, Kumar Saha S, Yang G, Choi H, Cho S: Recent Advances in Disease Modeling and Drug Discovery for Diabetes Mellitus Using Induced Pluripotent Stem Cells. Int J Mol Sci 2016;17:256.

2 Shaw J, Sicree R, Zimmet P: Global estimates of the prevalence of diabetes for 2010 and 2030. Diabetes Res Clin Pract 2010;87:4-14. 


\section{Cellular Physiology Cell Physiol Biochem 2018;49:123-133 \begin{tabular}{l|l|l} 
and Biochemistry Published online: 22 August, 2018 & $\begin{array}{l}\text { @ } 2018 \text { The Author(s). Published by S. Karger AG, Basel } \\
\text { www.karger.com/cpb }\end{array}$ \\
\hline
\end{tabular}}

Jiang et al.: CXCL13 Promoted the Fracture Healing in Diabetic Rats

3 Ko K, Coimbra L, Tian C, Alblowi J, Kayal R, Einhorn T, Gerstenfeld L, Pignolo R, Graves D: Diabetes reduces mesenchymal stem cells in fracture healing through a TNF $\alpha$-mediated mechanism. Diabetologia 2015;58:633-642.

4 Moseley K: Type 2 diabetes and bone fractures. Curr Opin Endocrinol Diabetes Obes 2012;19:128-135.

5 Roszer T: Inflammation as death or life signal in diabetic fracture healing. Inflamm Res 2011;60:3-10.

6 Menchine M, Probst M, Agy C, Bach D, Arora S: Diagnostic accuracy of venous blood gas electrolytes for identifying diabetic ketoacidosis in the emergency department. Acad Emerg Med 2011;18:1105-1108.

7 Hernigou P, Guissou I, Homma Y, Poignard A, Chevallier N, Rouard H, Flouzat Lachaniette C: Percutaneous injection of bone marrow mesenchymal stem cells for ankle non-unions decreases complications in patients with diabetes. Int Orthop 2015;39:1639-1643.

-8 Ono T, Takada S, Kinugawa S, Tsutsui H: Curcumin ameliorates skeletal muscle atrophy in type 1 diabetic mice by inhibiting protein ubiquitination. Exp Physiol 2015;100:1052-1063.

$>$ Sun M, Yang J, Wang J, Hao T, Jiang D, Bao G, Liu G: TNF- $\alpha$ is upregulated in T2DM patients with fracture and promotes the apoptosis of osteoblast cells in vitro in the presence of high glucose. Cytokine 2016;80:35-42.

10 Shariful Islam S, Lechner A, Ferrari U, Froeschl G, Niessen L, Seissler J, Alam D: Social and economic impact of diabetics in Bangladesh: protocol for a case-control study. BMC Public Health 2013;13:1217.

11 Abdel-Salam B: Modulatory effect of whey proteins in some cytokines involved in wound healing in male diabetic albino rats. Inflammation 2014;37:1616-1622.

12 Allione A, Di Gaetano C, Dani N, Barberio D, Sieri S, Krogh V, Matullo G: Anticoagulants used in plasma collection affect adipokine multiplexed measurements. Cytokine 2016;80:43-47.

13 Alblowi J, Tian C, Siqueira M, Kayal R, McKenzie E, Behl Y, Gerstenfeld L, Einhorn T, Graves D: Chemokine expression is upregulated in chondrocytes in diabetic fracture healing. Bone 2013;53:294-300.

-14 Zhou W, Yu L, Fan J, Wan B, Jiang T, Yin J, Huang Y, Li Q, Yin G, Hu Z: Endogenous Parathyroid Hormone Promotes Fracture Healing by Increasing Expression of BMPR2 through cAMP/PKA/CREB Pathway in Mice. Cell Physiol Biochem 2017;42:551-563.

15 Tang P, Duan C, Wang Z, Wang C, Meng G, Lin K, Yang Q, Yuan Z: NPY and CGRP Inhibitor Influence on ERK Pathway and Macrophage Aggregation during Fracture Healing. Cell Physiol Biochem 2017;41:1457-1467.

$>16$ Huang J, Peng J, Cao G, Lu S, Liu L, Li Z, Zhou M, Feng M, Shen H: Hypoxia-Induced MicroRNA-429 Promotes Differentiation of MC3T3-E1 Osteoblastic Cells by Mediating ZFPM2 Expression. Cell Physiol Biochem 2016;39:1177-1186.

17 Khang D, Choi J, Im Y, Kim Y, Jang J, Kang S, Nam T, Song J, Park J: Role of subnano-, nano- and submicronsurface features on osteoblast differentiation of bone marrow mesenchymal stem cells. Biomaterials 2012;33:5997-6007.

18 Tian F, Ji X, Xiao W, Wang B, Wang F: CXCL13 promotes the effect of bone marrow mesenchymal stem cells (MSCs) on tendon-bone healing in rats and in C3HIOT1/2 cells. Int J Mol Sci 2015;16:3178-3187.

19 Rui Y, Lui P, Lee Y, Chan K: Higher BMP receptor expression and BMP-2-induced osteogenic differentiation in tendon-derived stem cells compared with bone-marrow-derived mesenchymal stem cells. Int Orthop 2012;36:1099-1107.

-20 Nedoszytko B, Sokołowska-Wojdyło M, Ruckemann-Dziurdzińska K, Roszkiewicz J, Nowicki R: Chemokines and cytokines network in the pathogenesis of the inflammatory skin diseases: atopic dermatitis, psoriasis and skin mastocytosis. Postepy Dermatol Alergol 2014;31:84-91.

-21 Zhou S, Wang J, Chiang C, Sheng L, Li Q: Mechanical stretch upregulates SDF- $1 \alpha$ in skin tissue and induces migration of circulating bone marrow-derived stem cells into the expanded skin. Stem Cells 2013;31:27032713.

22 Tian F, Ji X, Xiao W, Wang B, Wang F: CXCL13 Promotes Osteogenic Differentiation of Mesenchymal Stem Cells by Inhibiting miR-23a Expression. Stem Cells Int 2015;2015:632305.

23 Granero-Moltó F, Myers T, Weis J, Longobardi L, Li T, Yan Y, Case N, Rubin J, Spagnoli A: Mesenchymal stem cells expressing insulin-like growth factor-I (MSCIGF) promote fracture healing and restore new bone formation in Irs1 knockout mice: analyses of MSCIGF autocrine and paracrine regenerative effects. Stem Cells 2011;29:1537-1548.

-24 Kasahara T, Imai S, Kojima H, Katagi M, Kimura H, Chan L, Matsusue Y: Malfunction of bone marrow-derived osteoclasts and the delay of bone fracture healing in diabetic mice. Bone 2010;47:617-625.

25 Hamann C, Kirschner S, Günther K, Hofbauer L: Bone, sweet bone--osteoporotic fractures in diabetes mellitus. Nat Rev Endocrinol 2012;8:297-305. 


\section{Cellular Physiology Cell Physiol Biochem 2018;49:123-133 \begin{tabular}{ll|l} 
DOI: 10.1159/000492848 & $\begin{array}{l}\text { O 2018 The Author(s). Published by S. Karger AG, Basel } \\
\text { www.karger.com/cpb }\end{array}$
\end{tabular}}

Jiang et al.: CXCL13 Promoted the Fracture Healing in Diabetic Rats

26 Motyl K, McCauley L, McCabe L: Amelioration of type I diabetes-induced osteoporosis by parathyroid hormone is associated with improved osteoblast survival. J Cell Physiol 2012;227:1326-1334.

27 Starup-Linde J, Vestergaard P: Management of endocrine disease: Diabetes and osteoporosis: cause for concern? Eur J Endocrinol 2015;173:R93-99.

28 Phipps M, Xu Y, Bellis S: Delivery of platelet-derived growth factor as a chemotactic factor for mesenchymal stem cells by bone-mimetic electrospun scaffolds. PLoS ONE 2012;7:e40831.

-29 Castro-Manrreza M, Montesinos J: Immunoregulation by mesenchymal stem cells: biological aspects and clinical applications. J Immunol Res 2015;2015:394917.

-30 Dreger T, Watson J, Akers W, Molligan J, Achilefu S, Schon L, Zhang Z: Intravenous application of CD271selected mesenchymal stem cells during fracture healing. J Orthop Trauma 2014;28:S15-19.

-31 Weinberg E, Maymon T, Weinreb M: AGEs induce caspase-mediated apoptosis of rat BMSCs via TNF $\alpha$ production and oxidative stress. J Mol Endocrinol 2014;52:67-76.

-32 Karnes J, Daffner S, Watkins C: Multiple roles of tumor necrosis factor-alpha in fracture healing. Bone 2015;78:87-93.

33 Kim K, Park S, Im G: Osteogenic differentiation and angiogenesis with cocultured adipose-derived stromal cells and bone marrow stromal cells. Biomaterials 2014;35:4792-4804.

34 Smith H, Whittall C, Weksler B, Middleton J: Chemokines stimulate bidirectional migration of human mesenchymal stem cells across bone marrow endothelial cells. Stem Cells Dev 2012;21:476-486.

-35 Tian F, Ji XL, Xiao WA, Wang B, Wang F: CXCL13 promotes the effect of bone marrow mesenchymal stem cells (MSCs) on tendon-bone healing in rats and in C3HIOT1/2 cells. Int J Mol Sci 2015;16:3178-3187.

-36 Lisignoli G, Toneguzzi S, Piacentini A, Cattini L, Lenti A, Tschon M, Cristino S, Grassi F, Facchini A: Human osteoblasts express functional CXC chemokine receptors 3 and 5: activation by their ligands, CXCL10 and CXCL13, significantly induces alkaline phosphatase and beta- $\mathrm{N}$-acetylhexosaminidase release. J Cell Physiol 2003;194:71-79.

-37 Schoeman M, Moester M, Oostlander A, Kaijzel E, Valstar E, Nelissen R, Löwik C, de Rooij K: Inhibition of GSK3 $\beta$ Stimulates BMP Signaling and Decreases SOST Expression Which Results in Enhanced Osteoblast Differentiation. J Cell Biochem 2015;116:2938-2946.

38 Gandolfi M, Ciapetti G, Taddei P, Perut F, Tinti A, Cardoso M, Van Meerbeek B, Prati C: Apatite formation on bioactive calcium-silicate cements for dentistry affects surface topography and human marrow stromal cells proliferation. Dent Mater 2010;26:974-992. 